\title{
Krankheitsbegriff und Geschichtlichkeit der Krankheit
}

\author{
Von Antoinette Stettler
}

I.

Ein nicht unbeträchtlicher Teil der Medizingeschichte ist mit dem Aufspüren von Krankheiten bei den verschiedensten Völkern zu den verschiedensten Zeiten an den verschiedensten Orten beschäftigt, wobei namentlich das 19. Jahrhundert Monumentalwerke der Geschichte der Krankheiten hinterließ, so daß wir heutzutage ohne weitere Hintergedanken von der Geschichte der Lepra, der Pest, der Kreislaufkrankheiten, des Diabetes mellitus usw. sprechen. Von der Geschichte der Krankheit im Sinne der Pathographie sei hier gänzlich abgesehen.

Der Ausdruck Geschichte ist doch nun wohl so zu verstehen, daß ein Phänomen dann Geschichte hat, wenn es einen Anfang, einen Verlauf und vielleicht ein Ende in der Zeit aufweisen kann. Geschichte einer Krankheit wäre demnach das Aufspüren der Anfänge, der Nachweis einer Entwicklung und möglicherweise des Aufhörens derselben.

Hier meldet sich aber ein Widerspruch: Krankheit ist nämlich so sehr mit dem menschlichen Dasein verbunden, daß sie uns auf den ersten Blick als zeitlos erscheint, das heißt, daß die Frage nach ihrer Geschichte recht eigentlich sinnlos wäre. Daß Krankheit geschichtslos sei, gilt wohl in dieser allgemeinen Formulierung, doch ist diese so unscharf, weil dann unter Krankheit nichts anderes als Unwohlsein, Unangepaßtheit oder Normveränderung verstanden werden darf. Insofern ist der Krankheitsbegriff auch kein ausschließlich medizinischer mehr, sondern er definiert sich auf diese Weise auch vom Individuum oder von der Gesellschaft her.

Trotzdem ist die Frage nach der Geschichte der Krankheit legitim, sobald man sich einer wie auch immer gearteten einzelnen Normveränderung, einem einzelnen Krankheitsbild, zuwendet. Eine erste Beobachtung, welche die Geschichtlichkeit von Krankheitsbildern nahelegt, ist in der biologischen Tatsache gegeben, daß der Verlauf namentlich von Infektionskrankheiten oft ausgeprägte zeitliche Schwankungen aufweist, z. B. in den letzten dreißig Jahren der Scharlach, die Diphtherie, die Grippe u. a.m. Dies kann vermuten lassen, daß solche plastischen, variablen Krankheitsbilder während der Menschheitsgeschichte einmal ihren Ursprung genommen haben, sich in ihrem Verlauf verändert haben und auch ohne therapeutisches Dazutun wie Impfkampagnen einmal verschwinden mögen oder verschwunden sind. Dabei ist man bis zum Anfang des 20. Jahrhun- 
derts weit davon entfernt, über den genauen Zeitpunkt und die Umstände des Kommens und Gehens orientiert zu sein. Immerhin ist es auffällig, daß Beschreibungen von Viruskrankheiten, so wie sie sich uns heute präsentieren, mit Ausnahme der Pocken und Masern, die Rhazes beschrieb, erst relativ spät, das heißt seit der Renaissance datieren. Um eine Viruskrankheit, die plötzlich kam und seither verschwunden ist, mag es sich bei dem in der Tudorzeit beschriebenen Schwitzfieber gehandelt haben. Das berühmteste Beispiel einer dergestalt neu auftretenden Krankheit ist gewiß die Syphilisepidemie des Jahres 1493 in Neapel.

Ferner liegt es tatsächlich auf der Hand, daß es beispielsweise toxische Krankheitsphänomene (Giftunfall von Seveso 1976) oder gewisse Mangelzustände (Skorbut der Seeleute auf langen Fahrten) gibt, die so in einer andern Zivilisation nicht auftreten könnten, daß es mithin Krankheitsbilder gibt, die Geschichte haben. Daraus resultieren die bekannten Spekulationen, daß Krankheit ausschließlich zivilisationsbedingt sei. Paradigmen der Zivilisationskrankheiten des heutigen Tages sind die psychosomatischen Erscheinungsbilder.

Schließlich wurde die Geschichtlichkeit von Krankheiten als prinzipielle Möglichkeit schon vom älteren Plinius in Betracht gezogen und in der Renaissance von Falloppio wieder aufgegriffen. Allein schon durch ihr Alter scheint die Frage nach der Geschichtlichkeit der Krankheiten gerechtfertigt. Bei der Beantwortung dieser Frage ist vom Krankheitsbegriff, von der Krankheitsdefinition, auszugehen, von der bekanntlich jedermann seine eigene Vorstellung hat.

II.

Es ist das Verdienst von Rothschuh, in diesen Wirrwarr eine Ordnung gebracht zu haben, indem er die Krankheitsbegriffe als Krankheitsmodelle systematisierte $^{1}$. Seine Systematik ist beispielhaft und so vollständig, wie man es nur wünschen kann. Rothschuh stellt dabei auch zur Diskussion, ob die Vielzahl der Krankheitsbegriffe nicht aus dem Sachverhalt bzw. aus dem relationellen Standpunkt des jeweiligen Betrachters gerechtfertigt und notwendig sei. Die Krankheitsbegriffe, die Rothschuh systematisiert, haben alle gemeinsam, daß von der Krankheit gesagt wird, sie sei wesentlich das und das, z. B. metaphysisch: Strafe Gottes; philosophisch-spekulativ: Säfteverderbnis; naturalistisch: Folge fehlerhafter Struktur der Gewebe usw.

Wenn von einem Phänomen, also von der Krankheit gesagt wird, sie sei wesentlich das und das, haben wir einen essentiell definierten Begriff vor uns. Krankheit, essentiell definiert, ist zwar vom jeweiligen Stande der naturkundlichen 
Realkenntnisse abhängig, aber eigentlich Glaubens- oder Ermessenssache und bleibt oft der Spekulation überlassen. Krankheit, essentiell definiert, ist nicht oder nur teilweise objektivierbar.

Hier ist einzuflechten, daß der sogenannte «ontologische» Krankheitsbegriff, dessen Geschichte Temkin als Gegenstück zum sogenannten «physiologischen» Krankheitsbegriff (Krankheit ist gleich functio laesa) beschrieben hat ${ }^{2}$, bloß eine sehr weit getriebene essentielle Auffassung des Krankheitsbegriffes darstellt. Überdies ist das Begriffspaar ontologisch - physiologisch nicht besonders glücklich gewählt. Wir möchten uns eher Probst anschließen, der diesen Unterschied in der Krankheitsauffassung im Anschluß an die hochmittelalterliche Scholastik «begriffsrealistisch» und «nominalistisch» nennt ${ }^{3}$. Selbstverständlich handelt es sich beim «begriffsrealistischen » wie auch beim «nominalistischen » Krankheitsbegriff um einen essentiell definierten Krankheitsbegriff.

III.

Der Krankheitsbegriff kann statt essentiell aber auch akzidentiell, das heißt durch Aufzählung der als geeignet sich darbietenden einzelnen Krankheitsbilder definiert werden. Krankheitsbild wäre in einem solchen Fall Tumor oder Pneumonie oder Migräne oder Lepra usw., je nach der Zeitepoche, die wir ins Auge fassen. Eine solche Aufzählung der akzidentiellen Krankheitsbegriffe wäre schier endlos, und es gibt deren heute offenbar an die 30000 . Daß sie sehr detailliert sind, braucht man nicht zu betonen.

Krankheit, akzidentiell bestimmt, ist - weil gestalthafte Wahrnehmung von Körper- oder Funktionsveränderung - objektivierbar.

Gibt es nun die Möglichkeit, die Geschichte der Krankheitsphänomene, der Krankheitsbilder, zu schreiben? Wenn sich auch hier die Frage nach dem Krankheitsbegriff stellt, so nicht nach dem in der geschilderten apodiktischen Art: «Krankheit ist wesentlich das und das», sondern nach demjenigen, der gemäß dem Idealbild der Pathologie geformt ist. Danach gehört zum Krankheitsbegriff neben der Symptomatologie die Ätiologie und die Pathogenese mit dem Ziel einer wirksamen Therapie.

Wenn wir nun von diesen unsern heutigen Krankheitsbildern ausgehen - und das müssen wir - und uns an die Verfolgung ebendieser Bilder in die Vergangenheit machen, sind wir mit zwei grundsätzlichen Hindernissen konfrontiert. Dem einen stehen alle Historiker gegenüber: es liegt in der Dürftigkeit der Quellen. Das zweite Hindernis ist vielleicht nur dem Historiker von Krankheiten eigen: es besteht im kaleidoskopartigen Wechsel dessen, was die einzelnen Zeitepochen 
als dem Krankheitsbild zugehörig bezeichnen, und dem, was wir dem Krankheitsbild zuschreiben. Das Hindernis besteht also im jeweils verschiedenen Umfang des Begriffes. Dazu tritt noch der wechselnde Stellenwert dessen, was jeweils als Symptom, Ursache oder Krankheitsfolge in einem bestimmten Krankheitsbild aufgefaßt wird.

Es ist zu befürchten, daß es nur einige wenige Krankheitsbilder gibt, die sich dergestalt völlig zur Deckung bringen lassen, von denen also mit Sicherheit gesagt werden kann, sie hätten so wie heute in einer früheren Zeit existiert.

Man könnte einwenden, es gereiche zur Zufriedenheit, wenn man wisse, daß die alten Ägypter schon vom Fieber geplagt, die alten Griechen schon den süßen Urin, den Hirnschlag usw. gekannt hätten, auch wenn die damaligen Ärzte Fieber für eine Krankheit hielten, während wir es als Symptom ansehen. Ebenso meinen wir heute den Mechanismus des Diabetes mellitus oder der Kreislaufkrankheiten zu kennen. Aber können wir denn wirklich bei derart wechselnden Krankheitsinterpretationen immer von ein und demselben Krankheitsbild reden ? Als Arbeitshypothese müssen wir das wohl zunächst annehmen, weil wir sonst gar keine Geschichte der Krankheiten schreiben könnten. Doch müssen wir uns dabei klar sein, daß die Geschichte einer Krankheit nicht so sehr die Geschichte ihrer realen Entwicklung ist als vielmehr die Geschichte der Begriffsausfüllung. Wir würden methodisch demnach so vorgehen, daß wir unsere heutigen Krankheitsbilder ihrer allermeisten Bestandteile entleeren, so daß gewissermaßen nur noch ein Rahmenbegriff, ein Umriß, stehenbliebe. Mit diesem «Gerät» würden wir dann an die Quellen herangehen, um zu sehen, was etwa da hineinpassen würde.

Man kann allerdings auch umgekehrt argumentieren, daß wir nämlich unsere heutigen detaillierten Krankheitsbilder gar nicht hätten, wenn diese Krankheitsbilder nicht schon in Umrissen seit Jahrhunderten bekannt wären und die Forschung zu immer detaillierteren Fragen angeregt hätten. Wenn dem so ist, hätten die früheren Krankheitsbeobachter eine geradezu phänomenale Intuition gehabt.

Es ist in der Frage der Geschichtsschreibung der Krankheiten ein mittlerer Standpunkt einzunehmen, der etwa dahin lautet: es ist denkbar und wahrscheinlich, daß es viele der heutigen Krankheitsbilder faktisch schon sehr lange und vielerorts gibt, daß wir aber bei der historischen Betrachtung nicht in erster Linie zu konstatieren vermögen, wie, wann und unter welchen Bedingungen diese Krankheitsbilder entstanden sind, sondern nur nachweisen können, wie und in welcher Weise sich ein bestimmter akzidentieller Krankheitsbegriff füllte oder entleerte (siehe gewisse humoral-pathologische Krankheitsbilder), wobei wir auch dann darauf achten müssen, nicht über die so wechselhafte Vielzahl der akzidentiellen Krankheitsbegriffe zu stolpern. 


\section{Anmerkungen}

${ }^{1}$ Karl Eduard Rothschuh, Der Krankheitsbegriff (Was ist Krankheit?), in: Was ist Krankheit?, hrsg. von K.E. Rothschuh, Darmstadt 1975, S. 397-421.

${ }^{2}$ Owsei Temkin, The Scientific Approach to Disease, in: Scientific Change. Symposion on the History of Science (ed. E. Crombie). New York/London 1963, S. 629-647.

${ }^{3}$ Christian Probst, Der Weg des ärztlichen Erkennens am Krankenbett. In: Sudhoffs Archiv, Beiheft 15, 1972.

Frau Dr.med.Antoinette Stettler

Hallwylstraße 24.

3005 Bern 